\title{
Single user tracing superimposed codes
}

\author{
Miklós Csürös ${ }^{1}$ \\ Dép. Informatique et recherche \\ opérationnelle, Université de Montréal \\ CP 6128 succ. Centre-Ville, \\ Montréal, Qué. H3C 3J7, Canada \\ Email: csuros@iro.umontreal.ca.
}

\author{
Miklós Ruszinkó2 \\ Computer and Automation Research \\ Institute, Hungarian Academy of Sciences \\ Budapest, P. O. Box 63, Hungary-1518 \\ Email: ruszinko@lutra.sztaki.hu.
}

\begin{abstract}
We investigate the zero-error capacity region of $r$-out of $T$ user multiple access $O R$ channel. A family $\mathcal{F}$ subsets of $[n]=\{1, \ldots, n\}$ is an $r$-single-usertracing superimposed code ( $r$-SUT) if there exists such a single-user-tracing function $\phi: 2^{[n]} \mapsto \mathcal{F}$ that for all $\mathcal{F}^{\prime} \subseteq \mathcal{F}$ with $\left|\mathcal{F}^{\prime}\right| \leq r, \phi\left(\cup_{A \in \mathcal{F}^{\prime}} A\right) \in \mathcal{F}^{\prime}$. In this paper we introduce the concept of these codes and investigate bounds on their rate. We also consider disjointly $r$ superimposed codes.
\end{abstract}

\section{SinglE-USER-TRACING SUPERIMPOSED CODES}

Suppose that $T$ users share a common channel. To each of them a binary vector of length $n$ is associated. The $i^{\text {th }}$ user transmits its vector $x_{i}=\left(x_{i}^{1}, \ldots, x_{i}^{n}\right)(i=1,2, \ldots, T)$ if it is active, otherwise not. It is assumed that the transmission is bit and block synchronized. The destination of the messages is a single receiver, which gets the bitwise $O R$ vector of the vectors associated to the active users. Moreover, suppose that at most $r$ users are active simultaneously. In the classical framework of superimposed coding, the receiver has to be able to identify the set of all active users from the output vector $\mathbf{y}$ of the channel. That is, the code must satisfy the property that for all choices of $x_{1}, \ldots, x_{k}$ and $z_{1}, \ldots, z_{\ell}$ of codewords with $1 \leq k, \ell \leq r$ and $\left\{x_{1}, \ldots, x_{k}\right\} \neq\left\{z_{1}, \ldots, z_{\ell}\right\}$, we have $\vee_{i=1}^{k} x_{i} \not \neq \vee_{j=1}^{\ell} z_{j}$. The rate of these codes have been studied extensively in e.g., [2, 3, 4].

Here we investigate the case when the receiver has to be able to identify just one user out of at most $r$ active ones. Clearly, if a code is superimposed in the classical sense then it satisfies this requirement. A practical motivation for our study of $r$-SUT families rises from a novel application of combinatorial designs in genomics [1].

As the question is rather of a combinatorial nature, we switch to that terminology. Codewords are characteristic vectors of subsets of the set $[n]=\{1, \ldots, n\}$ with $n>0$.

Definition I.1 $A$ family $\mathcal{F} \subseteq 2^{[n]}$ is r-superimposed if $\bigcup_{i=1}^{k} A_{i} \neq \bigcup_{j=1}^{\ell} B_{i}$ for any $\left\{\bar{A}_{1}, \ldots, A_{k}\right\} \neq\left\{B_{1}, \ldots, B_{\ell}\right\}$, $1 \leq k, \ell \leq r ; A_{1}, \ldots, A_{k}, B_{1}, \ldots, B_{\ell} \in \mathcal{F}$.

We are interested in $r$-single-user-tracing families ( $r$-SUT), defined as follows.

Definition I.2 A family $\mathcal{F}$ is $r$-SUT if for all choices of $\mathcal{F}_{1}, \ldots, \mathcal{F}_{k} \subseteq \mathcal{F}$ with $\left|\mathcal{F}_{i}\right| \leq r, \bigcup_{A \in \mathcal{F}_{1}} A=\bigcup_{A \in \mathcal{F}_{2}} A=\ldots=$

\footnotetext{
${ }^{1}$ Research supported by NSERC Grant 250391-02.

${ }^{2}$ Research supported in part by OTKA Grants T038198, T046234. This work relates to Department of the Navy Grant N00014-04-1-4034 issued by the Office of Naval Research International Field Office. The United States Government has a royaltyfree license throughout the world in all copyrightable material contained herein.
}

$\bigcup_{A \in \mathcal{F}_{k}} A$ implies $\cap_{i=1}^{k} \mathcal{F}_{k} \neq \emptyset$. Equivalently, there exists such a single-user-tracing function $\phi: 2^{[n]} \mapsto \mathcal{F}$ that for all $\mathcal{F}^{\prime} \subseteq \mathcal{F}$ with $\left|\mathcal{F}^{\prime}\right| \leq r, \phi\left(\cup_{A \in \mathcal{F}^{\prime}} A\right) \in \mathcal{F}^{\prime}$.

For every base set size $n$ and $r$, let $f(n, r)$ denote the maximum size of an $r$-superimposed family, and $g(n, r)$ denote the maximum size of an $r$-SUT family. We give bounds on the rate of $r$-SUT families, which is $R_{g}(r)=\limsup _{n \rightarrow \infty} \frac{\log g(n, r)}{n}$.

Theorem I.3 $\exists c_{1}, c_{2}>0$ such that $\frac{c_{1}}{r^{2}} \leq R_{g}(r) \leq \frac{c_{2}}{r}$.

\section{DISJOINTLY $r$-SUPERIMPOSED CODES}

Another important case implicated in the multiple access model of Section 1 is when the receiver must distinguish only between disjoint sets of active users. The following definition captures this notion.

Definition II.1 $\mathcal{F} \subseteq 2^{[n]}$ is disjointly $r$-superimposed if $\bigcup_{i=1}^{k} A_{i} \neq \bigcup_{j=1}^{\ell} B_{i}$ for $\left\{A_{1}, A_{2}, \ldots, A_{k}\right\} \cap\left\{B_{1}, B_{2}, \ldots, B_{\ell}\right\}=$ $\emptyset ; 1 \leq k, \ell \leq r ; A_{1}, \ldots, A_{k}, B_{1}, \ldots, B_{\ell} \in \mathcal{F}$.

Let $h(n, r)$ be the maximum size of disjointly $r$-superimposed families.

Lemma II.2 If $\mathcal{F}$ is $r$-superimposed then it is $r$-SUT. If $\mathcal{F}$ is $r$-SUT, then it is disjointly $r$-superimposed. Hence, $f(n, r) \leq$ $g(n, r) \leq h(n, r)$.

Despite the seemingly slight difference between Definitions II.1 and I.1, the extremal properties of disjointly $r$ superimposed codes (families) and $r$-superimposed ones differ substantially.

Theorem II.3 $\exists c_{1}, c_{2}>0$ such that $\frac{c_{1}}{r} \leq R_{h}(r) \leq \frac{c_{2} \log r}{r}$.

\section{ACKNOWLEDGMENTS}

We would like to thank Noga Alon and László Györfi for fruitful discussions.

\section{REFERENCES}

[1] M. Csürös and A. Milosavljevic. Pooled Genomic Indexing (PGI): mathematical analysis and experiment design. In Proc. Workshop on Algorithms in Bioinformatics, pp. 10-28, NY: Springer-Verlag 2002.

[2] A. G. D'yachkov and V. V. Rykov. Bounds on the length of disjunctive codes. Problemy Peredachi Informatsii vol. 18, no. 3, pp. 7-13, 1982.

[3] W. H. Kautz and R. C. Singleton. Nonrandom binary superimposed codes. IEEE Trans. Inform. Theory, vol. IT-10, pp. 363-377, October 1964.

[4] M. Ruszinkó. On the upper bound of the size of the $r$-cover-free families. Journal of Combinatorial Theory, Series A vol. 66, no. 2, pp. 302-310, May 1994. 